\title{
Ernst Seidl, Frank Loose und Edgar Bierende (Hrsg.): Mathematik mit Modellen
}

\section{Universität Tübingen, 2018, 399 Seiten mit sehr vielen Bildern, ISBN 978-3-9819182-0-5, 34,90€}

\section{Klaus Volkert}

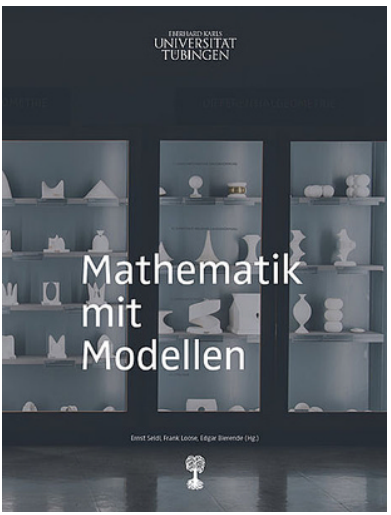

Mathematische Modelle hatten in den letzten Jahren ohne Zweifel Konjunktur, wie auch mehrere Artikel in den „Semesterberichten“ belegen. ${ }^{1}$ Wurden vor 50 Jahren Assistentinnen und Assistenten damit beauftragt, diese verstaubten Objekte zu entsorgen, so schwärmten sie in den letzten Jahren aus, um solche in Kellern und Speichern wieder aufzustöbern. Die mehr oder minder zahlreichen Fundstücke wurden restauriert, fotografiert und im Internet präsentiert.

\footnotetext{
1 Mehrere interessante Beiträge zum Thema „mathematische Modelle“ enthält auch die Nummer 2 (2017) des „Mathematical Intelligencer“.

K. Volkert $(\bowtie)$

AG Didaktik und Geschichte der Mathematik, Bergische Universität Wuppertal, Wuppertal, Deutschland

E-Mail: klaus.volkert@math.uni-wuppertal.de
} 
So geschehen auch in Tübingen. Allerdings hatte man dort den Vorteil, dass hier mit Alexander von² Brill (1842-1935) von 1884 bis 1918 ein wichtiger Befürworter und Produzent mathematischer Modelle wirkte, der zudem durch den Verlag seines Bruders Ludwig in Darmstadt maßgeblich an deren Vermarktung beteiligt war. Wenn im Folgenden von Brillschen Modellen die Rede ist, so sind damit meist solche gemeint, die diese Firma vertrieb. Von der Reichhaltigkeit des Angebots $^{3}$ dieser Firma zeugt der zweite Teil des hier zu besprechenden Buches. Viele der von Bruder Ludwig verkauften Modelle gingen auf Alexander Brill zurück - aber bei weitem nicht alle. Die Ausbeute des Suchens fiel in Tübingen sehr reichhaltig aus. Das vorliegende Buch, ein aufgrund der vielen oft farbigen Abbildungen prächtiger und schwergewichtiger Band, behandelt die Tübinger Modellsammlung anlässlich ihrer Präsentation in der Öffentlichkeit aus verschiedenen Perspektiven. Dokumentiert werden insbesondere auch Arbeiten von Studierenden, die sich in ihrem Praxissemester im Rahmen eines Master-Profils „Museen \& Sammlungen“ mit diesen Modellen beschäftigten. Es zeigte sich, dass diese Modelle auch heute noch eine große Anziehungskraft besitzen und dass sie nichts von ihrem didaktischen Wert eingebüßt haben. Somit haben wir hier auch eine gelungene hochschuldidaktische Anregung vor uns.

Welche Modelle sind aber eigentlich gemeint? Es geht hier um materielle Objekte, die abstrakte mathematische Entitäten - wie Körper, Flächen, Kurven oder funktionale Zusammenhänge - verdeutlichen sollen oder wie es zu Beginn des Buches „Mathematik mit Modellen“ heißt: „Sie materialisieren ein immaterielles Gedankenkonstrukt, ...“ (p. 20)4 . Einfache Objekte dieser Art, wie Würfel (ein besonders schönes Beispiel findet sich auf p. 315) oder Dodekaeder (vgl. p. 137), waren wohl schon immer in Gebrauch, u.a. zu Alltagszwecken (z. B. Spielzeug) und als Veranschaulichungen. Insbesondere spielten Modelle eine wichtige Rolle als Vorlagen fürs Zeichnen, etwa im Rahmen von Konstruktionsübungen. ${ }^{5}$ In Karlsruhe legte schon Guido Schreiber, der hier von 1827 bis 1854 darstellende und praktische Geometrie am Polytechnikum lehrte, als solcher Vorgänger von Christian Wiener (siehe unten), am Polytechnikum eine Modellsammlung mit zwanzig Nummern an.

Das Buch „Mathematik mit Modellen“, dessen Kernstück die Modelle ausmachen, die in der Dauerausstellung „Mind and Shape“ in Tübingen zu sehen sind, gliedert sich in zwei große Teile überschrieben mit „Beiträge“ und „Modelle und Modellserien“. Die „Beiträge“ bringen mehrere im Schnitt etwa zehnseitige Artikel rund um das Thema des Buches, beginnend mit der Einleitung von E. Seidl, Direktor des Tübinger Universitätsmuseums, die die Themen des Buches umreißt, das zugrundeliegende studentische Projekt schildert und die Zentralfigur Alexander von Brill einführt. Weiter geht es mit einer durchaus nicht nur für Fachleute gedachten Einfüh-

\footnotetext{
2 „Von“ seit 1897. Nobilierungen von Mathematikern gab es bis zum Ende des Kaiserreiches gelegentlich in Süddeutschland, weitere Beispiele neben Brill sind F. Lindemann und W. Dyck.

3 Nach mehreren Stellen des Buches finden sich Abbildungen von Anzeigen und von Seiten aus Katalogen der Firma Brill bzw. ihres Nachfolgers (ab 1899) Friedrich Schilling in Halle; vgl. etwa pp. 213-215.

4 Seitenzahlen ohne weitere Angaben beziehen sich immer auf das hier zu besprechende Buch.

5 Zum Thema Zeichnen allgemein und v. a. im technisch-gewerblichen Kontext und seine Wichtigkeit vgl. man Klinger [8], König [9] und Lipsmeier [12].
} 
rung zur Mathematik der Modelle von F. Loose, die sich auf bahnbrechende Ideen der Geometrie, insbesondere auf Symmetrie, konzentriert, und einer „Geschichte der mathematischen Modelle“ des Tübinger Mathematikhistorikers und Ortskenners G. Betsch. Es folgen Beiträge zu den Modellausstellungen in München (siehe unten) und Chicago (beide 1893) von E. Bierende und F. Loose, bei denen natürlich auch Brillsche Modelle präsentiert wurden, und ein Artikel von E. Bierende über A. Brill als Sammler, Ideengeber und Initiator von Modellen, sowie ein kurzer Bericht zu den Irrfahrten ${ }^{6}$ der Tübinger Modellsammlung mit dem hübschen Titel „Pedelle und Modelle“ von S. Stangler und ein Interview mit der Tübinger Mathematikerin Carla Cederbaum zur heutigen Bedeutung und Verwendung von Modellen. Den Abschluss dieses Teiles bilden Betrachtungen zu mathematischen Modellen als Phänomene der Kunst von E. Seidl. Das bekannteste Beispiel hierfür sind vielleicht die Fotografien von Man Ray, die dieser in den 1930er Jahren von Modellen im Pariser Institut Henri Poincaré gemacht hat. In diesem ersten Teil finden sich zudem verschiedene nützliche Informationen wie Lebenslauf und Publikationsliste von Brill sowie ein Verzeichnis zu den wichtigsten Modellausstellungen. Der zweite Teil bringt Arbeiten von Studierenden zu verschiedenen Serien der Brillschen Modellsammlung, zu Christian Wiener, einem der prominentesten Modellbauer in seiner Zeit, und seinem Sohn Hermann, zu Julius Plücker und Felix Klein, aber auch zu weniger bekannten bis unbekannten Personen, die zur Brillschen Sammlung durch Bau (von Prototypen [,Urmodelle“]) von Modellen Beiträge geleistet haben. Hervorzuheben ist der Artikel über den französischen Modellbauer Charles Muret (pp. 158-167), über den bislang in Deutschland nur wenig bekannt war. Etwa die Hälfte aller Seiten des Buches bieten Fotografien von hoher Qualität dar; sie machen das visuelle Zentrum dieses Werkes aus und unterstreichen eindrucksvoll die ästhetische Dimension des Themas. Neben Modellen finden sich auch Geschäftsannoncen, Beschreibungen, Seiten aus Ausgabenbüchern, Inventarlisten und vieles andere mehr - eine gelungene und authentische Abrundung der Informationen. Hier wird viel neues Material geboten.

Da im vorliegenden Werk viele Themen an mehreren Stellen angesprochen werden, wird im Folgenden eine thematisch orientierte Darstellung zu einigen Aspekten gegeben. Angesichts der Vielfalt der Themen im Buch können hier nur einige Punkte herausgegriffen werden, eine erschöpfende Diskussion ist im hier gebotenen Rahmen leider nicht möglich.

Modelle können mindestens drei Funktionen haben: Sie dienen als Objekte der Forschung, der Lehre und der Repräsentation - wobei natürlich Mischformen möglich sind. ${ }^{7}$ Alle drei Aspekte kommen im vorliegenden Werk vor. Modelle als Forschungsgegenstand begegnen beispielsweise in der Beschreibung, die Christian Wiener von den Grundlagen gegeben hat, auf der seine Konstruktion (1868) des Modells eines Fläche dritter Ordnung mit 27 reellen Geraden beruhte (pp. 187-188). Dabei spielte die Schläflische Doppelsechs eine wichtige Rolle. Ein Modell der fraglichen

\footnotetext{
${ }^{6}$ Über einen ähnlichen Vorgang in Heidelberg, der sogar zum Verlust der Sammlung führte, berichtet Etwein [4].

${ }^{7}$ Eine ausführlichere Diskussion möglicher begrifflicher Klassifikationen von Modellen findet sich in der von E. Seidl verfassten Einleitung des Bandes (pp. 19-22).
} 
Fläche war übrigens schon vor Wiener von W. Fiedler um 1865 herum in Prag mit Hilfe seines Assistenten Rafael Morstadt erstellt worden, insofern ist das Zitat von Brill (p. 193 n. 15) zu relativieren. ${ }^{8}$ Allerdings versuchte Fiedler nicht, sein aus verlöteten Metallstäben bestehendes Modell zu vermarkten, während Wiener sich entschied, sowohl sein aus Gips gefertigtes Modell, aber auch preisgünstige stereoskopische Fotografien desselben zum Verkauf anzubieten. Beispiele solcher Fotos finden sich im vorliegenden Band auf p. 191; es gehören immer zwei fast identische Fotos zusammen, diese werden in einen speziellen Betrachter eingelegt und liefern dann durch Überlagerung den gewünschten räumlichen Effekt. Wiener, Professor der darstellenden Geometrie am Polytechnikum in Karlsruhe, ist ein eigener Abschnitt im Buch gewidmet (p. 186-193). Er war ein Pionier des Modellbaus und -vertriebs in Deutschland, Onkel und zeitweise auch Lehrer von Brill. Da Flächen dritter Ordnung, insbesondere solche mit 27 reellen Geraden, seinerzeit ein aktueller und vielbeachteter Forschungsgegenstand waren, war ein Modell natürlich willkommen (pp. 187-188) ${ }^{9}$. Weitgehend verdrängt wurde Wieners Modell durch eines der Clebschen Diagonalfläche, da dieses übersichtlicher und symmetrischer war. Es wurde von Adolf Weiler gebaut, Schüler von Fiedler, der sich nach Abschluss seines Studiums am Züricher Polytechnikum 1872 in Göttingen aufhielt (Volkert [15]), um dann Klein nach Erlangen zu folgen und dort zu promovieren. Weiler berichtete brieflich seinem Lehrer Fiedler über das Mathematiker-Treffen 1873 in Göttingen, das - angeregt durch den überraschend verstorbenen Alfred Clebsch die Gründung einer Mathematiker-Vereinigung ${ }^{10}$ vorbereiten sollte und auch eine Modellsammlung umfasste:

Die folgenden Tage hatten alle ungefähr denselben Charakter: Auf Spaziergängen \& bei den Modellen lernte man sich kennen: jeder konnte leicht zu jedem andern gelangen. Vorträge fanden kaum statt. Alles war sehr gut angeordnet, auch das Wetter war gut, [...]. Neben den anwesenden Leuten interessierten mich vor allem die Modelle von Eigel in Bonn [...], die Modelle von Herrn Prof. Schwarz der Minimalflächen gefallen allgemein sehr gut. ${ }^{11}$

Die Modelle waren also (auch) ein Treffpunkt - ein „Hotspot“ würde man heute sagen - und hatten somit auch eine kommunikative Funktion.

Wiener, der sehr breite bis ins Philosophische (z. B. Willensfreiheit) gehende Interessen hatte, spielte eine aktive Rolle in der Sektion für Mathematik (meist auch

\footnotetext{
8 Vgl. Fiedler [5].

9 Vgl. Lê [11] und Labs [10].

10 Vgl. Tobies-Volkert [14, S. 127-134]. Die Société Mathématique de France hatte sich 1872 in der Nachfolge des Deutsch-Französischen Krieges gegründet.

11 ETH-Bibliothek, Hochschularchiv, Hs 87: 1495, Brief von Weiler aus Göttingen an Fiedler vom 20. April 1873. Die Firma Johann Eigel Sohn (eigentlich in Köln, später wurden die Modelle von W. Lesemeister (ebenfalls Köln) übernommen) produzierte und vertrieb Modelle nach J. Plücker und F. Klein; vgl. Rowe [13]. David Rowe hat sich in den letzten Jahren in mehreren Arbeiten mit dem Thema Modelle als Forschungsmittel beschäftigt. Beispiele von Modellen nach Plücker und Klein sowie eine Werbung der Firma Lesemeister finden sich pp. 194-199 im Artikel über Klein. H. A. Schwarz war von 1870 bis 75 am Polytechnikum Zürich tätig. Er beschäftigte sich u.a. mit Minimalflächen, von denen er auch Modelle anfertigte. Diese wurden aber nicht kommerziell vertrieben; vgl. Brill [1, S. 74].
} 
für Physik oder Astronomie) der Versammlung deutscher Naturforscher und Ärzte. Anlässlich der Gießener Versammlung hatte er schon 1864 Modelle von Polyedern, vermutlich ging es um die Kepler-Poinsot'schen Sternpolyeder (vgl. pp. 54-55 und pp. 168-173), vorgestellt. Nach seinem eigenen Bericht wurde er 1867 bei der Versammlung in Frankfurt von A. Clebsch und anderen Sektionsmitgliedern aufgefordert, ein Modell der Fläche mit 27 reellen Geraden zu bauen, das er schon im nachfolgenden Jahr in Dresden präsentierte. Bei dieser Versammlung sprach übrigens Ernst Mach (Prag) über die Verwendung stereoskopischer Bilder im Unterricht (der Physik), von der er sich wichtige Verbesserungen erhoffte. Wiener war also nicht allein mit seinen Ideen. Auch später noch wurden bei den Versammlungen deutscher Naturforscher und Ärzte, bei denen ja die Mathematiker nur eine kleine Minderheit bildeten, gerne Modelle präsentiert ${ }^{12}$, was auf die dritte der oben genannten Funktionen, auf die Repräsentation, hinweist. Es ist also nicht erstaunlich, dass die Versammlung im vorliegenden Buch mehrmals vorkommt; aus ihrer mathematischen Sektion ging dann 1890 die Deutsche Mathematiker Vereinigung hervor. Diese sollte ihre Jahresversammlung nach wie vor im Rahmen der Versammlung deutscher Naturforscher und Ärzte abhalten. Schon für das für 1892 in Nürnberg geplante Treffen hatte man 1891 eine ,,umfassende Ausstellung mathematischer Modelle“13 geplant sicher auch mit dem Gedanken, die eigene Sichtbarkeit zu erhöhen. Aufgrund einer Cholera-Epidemie musste die Tagung 1892 abgesagt werden. ${ }^{14}$ Ihr Katalog erschien dennoch 1892, ein Nachtrag dazu 1893. Die Ausstellung selbst sowie ausnahmsweise die Versammlung der DMV wurden im September 1893 in München nachgeholt, wo Walter Dyck eine beachtliche Sammlung von Modellen in recht pompöser Inszenierung präsentieren konnte; vgl. hierzu pp. 93-96. Interessant, aber wohl den Rahmen des Buches sprengend, ist die Rolle mathematischer Modelle bei großen Ausstellungen wie Weltausstellungen, Gewerbeausstellungen etc., die sich an ein breites Publikum wandten. Die zweite Hälfte des 19. Jhs. war ja eine Hochzeit solcher Präsentationen ${ }^{15}$, die vermutlich nicht unwesentlich dazu beitrugen, das Bild der Mathematik in der Öffentlichkeit zu prägen.

Eine zweite forschende Verwendung von Modellen, die im vorliegenden Band erwähnt wird, bezieht sich auf die so genannte Kuensche Fläche (pp. 79-80), deren Natur als Ennepersche Fläche, also als einer Fläche, bei der durch jeden Punkt eine ebene Krümmungslinie geht, von Theodor Kuen anhand eines Modelles erkannt wurde. Damit komplettierte Kuen die Klassifikation der Enneperschen Flächen konstanter Gaußscher Krümmung. ${ }^{16}$

Die didaktische Verwendung von Modellen, also ihr Einsatz in der Lehre, schimmert an vielen Stellen des vorliegenden Werkes durch, allerdings ohne konkrete Hinweise, wie das geschehen ist. Auch Brills Diktum, ihm seien die Modelle in

\footnotetext{
12 Für eine Übersicht vgl. Tobies-Volkert [14, pp. 87-90].

13 Verhandlungen VdNÄ Halle 1891, 18. Die zeitlichen Angaben auf p. 87 sind unzutreffend.

14 Das gab es also schon vor Corona.

15 Vgl. Geppert [7]. Dank an Volker Remmert für den Hinweis auf diese Publikation.

16 Dank an F. Loose (Tübingen) für seine sehr hilfreichen Erklärungen zur Kuensche Fläche.
} 
der Lehre unentbehrlich geworden ${ }^{17}$, gibt hierüber leider keine Aufschlüsse. Natürlich muss man auch die Konstruktion von Modellen durch Studierende, wie sie im zweiten Teil des Buches an mehreren Beispielen vorgeführt wird, eine didaktische Verwendung nennen. Diese gab insbesondere Anlass zu produktivem Austausch zwischen Lehrendem und Lernenden.

Eine interessante Frage ist die nach den für die Modelle verwandten Materialien - besonders dann, wenn man an eine umfassende „Objektwissenschaft“ denkt (vgl. p. 147) oder von den Medienwissenschaften kommt und sich nur der ,material culture“" widmen möchte. Ein Großteil der Brillschen Modelle sind in Gips gefertigt, was Übung im Umgang mit diesem nicht einfach zu handhabenden Material erforderte. A. Brill und Kollege F. Klein hatten den Vorteil, in München ${ }^{18}$ mit J. Kreittmayr einen erfahrenen, am K. G. Nationalmuseum tätigen Formator in Gips vor Ort vorzufinden, der für die abzugießenden Urmodelle sorgte. ${ }^{19}$ Schon Christian Wiener hatte sein Modell einer Fläche dritter Ordnung mit 27 Geraden in Gips gefertigt; dieses recht haltbare Material eignete sich gut für serienmäßige Produktion und für den Vertrieb. Es hatte aber durchaus auch Nachteile, so konnte man ja nur die Oberfläche sehen und oft diese auch nicht komplett; zudem waren die Modelle starr. Dies war anders bei Modellen aus Fäden und Stangen, die man zumindest in gewissen Grenzen beweglich gestalten konnte. Vor allem Fäden eigneten sich gut, um die Struktur von Regelflächen zu verdeutlichen, etwa eines geraden Kreiszylinders. Gestaltete man einen Kreis des Zylinders noch drehbar, so konnte man den Übergang zum einschaligen Hyperboloid mit seinen beiden geraden Erzeugendenscharen verdeutlichen, im Grenzfall sogar zum Doppelkegel. Ein solches Modell hatte schon Th. Olivier gebaut; Regelflächen spielten bei G. Monge und seinen Schülern und Nachfolgern eine wichtige Rolle - zum einen aus mathematischen Gründen (eine passende Theorie ließ sich mit den damals vorhandenen Mitteln entwickeln), zum andern aber auch aus praktischen (Erzeugung von Oberflächen durch Abtragen von Material vermöge einer Schneide, Darstellung durch Projektionen im Sinne der darstellenden Geometrie etwa zur konstruktiven Bestimmung von Durchdringungskurven). Beziehungen von Fadenmodellen zum modernen Konstruktivismus eines Noam Gabo sind offensichtlich (p. 144). Der Nachteil von Fadenmodellen liegt auf der Hand: Sie sind empfindlich, wenig alterungsbeständig und schnell defekt. Diese Nachteile haben Stabmodelle nicht. Allerdings ist es schwierig, Beweglichkeit in ihrem Fall zu erreichen. Hermann Wiener, der Sohn von Christian, Professor für darstellende Geometrie an der Technischen Hochschule Darmstadt, entwickelte hierfür ein besonderes Scharnier, das geschränkte Verbindungsgelenk (p. 337 vgl. auch das bewegliche einschalige Hyperboloid von Herrmann Wiener p. 341). Stabmodelle lassen sich gut projizieren (vgl. das Modell der Kugel mit Großkreisen

\footnotetext{
17 „Die Modelle stehen bei den meisten Geometern, die sie haben, in Kästen und Schränken, ohne daß sie in den Vorlesungen angewendet und verstanden werden. Bei mir bilden die unter meiner Leitung entstandenen ein lebendiges Glied der Vorlesungen über Flächenkrümmung und Raumgeometrie." (Brill [3, S. 110]).

18 Brill war von 1875 bis 1884 in München, Klein von 1875 bis 1880 . Beide wechselten dann an Universitäten (Tübingen bzw. Leipzig).

19 Vgl. pp. 109. Polytechnika verfügten stets über diverse Modellierwerkstätten, einen Fachmann zu finden war hier nicht schwierig.
} 
von $\mathrm{H}$. Wiener p. 336), so ergibt sich eine direkte Verbindung zur darstellenden und projektiven Geometrie. Sehr eindrücklich zeigen dies die Modelle von Raumkurven von Christian Wiener (pp. 282-283). Ein Nachteil sowohl von Faden- wie auch von Stabmodellen ist, dass sie keine zusammenhängenden Flächen, sondern nur einzelne isolierte Kurve in derselben erzeugen; Betrachterin und Betrachter müssen sich diese vielmehr vorstellen können. Schließlich eignen sie sich weniger für eine Serienproduktion, das Verfahren Urmodell und Abguss ist nicht möglich.

Alexander Brill begann seine Tätigkeit als Modellbauer mit Kartonmodellen (pp. 17-18), nach eigener Aussage angeregt von den Modelle O. Henrici's, die er beim Treffen 1873 in Göttingen sah. ${ }^{20}$ Dabei werden aus Karton Teile ausgeschnitten und in einander gesteckt. Das Material war billig, fast überall verfügbar, leicht transportabel und mit gängigen Hilfsmitteln zu bearbeiten; die Schnittbögen konnten per Druck einfach vervielfältigt werden. Die Modelle selbst waren jedoch fragil und gerieten vermutlich oft nicht so, wie man es sich wünschte. Zudem entsteht auch bei dieser Art von Kartonmodellen ebenso wie die Stabmodelle keine kontinuierliche Oberfläche, komplexe Objekte wie die Fläche dritter Ordnung mit 27 reellen Geraden lassen sich so kaum herstellen. Eine andere Art von Kartonmodell findet sich im vorliegenden Werk in Gestalt von Polyedermodellen, gefaltet oder geklebt aus Papier oder Karton (pp. 52-56, 130-131, 168-171, 354-359). A. Weiler hatte schon 1872 ein Ad-hoc-Modell mit Pappe gebaut: „Letzte Woche sprach ich im Colloquium über Regelflächen \& verfertigte dazu Modelle. Diese waren ziemlich klein \& bestanden aus Zigarrenkistchen, in denen Blätter in Löchern steckten \& dazwischen Fäden durchgezogen. "21

Die Beantwortung der Frage, Welches Material für welches Modell?, hängt also von mehreren Faktoren ab. Deshalb greift eine Antwort nur aus der Sicht des verwendeten Materials zu kurz. Es geht vielmehr immer auch um mathematische und didaktische Aspekte, manchmal natürlich auch um ökonomische. Die Dominanz der Gipsmodelle unter denjenigen, die heute in Deutschland noch verfügbar sind, dürfte auch auf deren Haltbarkeit und die vergleichsweise hohen Anschaffungskosten zurückzuführen sein. Was für Geld teuer gekauft worden war und Eingang ins Inventar gefunden hatte, wird nicht so schnell entsorgt wie ein privat hergestelltes kostenloses oder günstig erstandenes Exemplar. Interessant aber bislang kaum erforscht ist die Frage, inwiefern hier nationale Besonderheiten, Stile, einen Einfluss hatten - etwa im Sinne der Dominanz von Fadenmodellen in Frankreich bedingt durch den großen Einfluss von Monge und seiner Schule.

Eine spezielle Art von Modell taucht im Buch mehrmals auf. Es handelt sich um die so genannte Reliefperspektive, bei der es darum geht, eine räumliche Situation in perspektivischer Sicht räumlich darzustellen - wie das etwa bei Reliefs und Bühnenbildern der Fall ist. Angeregt von J. V. Poncelet (1822) war dies im 19. Jh. unter darstellenden Geometern ein mehrfach bearbeitetes Forschungsgebiet, dem auch Monographien (z. B. von R. Staudigl (1868) und L. Burmester (1883)) gewidmet wurden. Solche Modelle - das war übrigens in diesem Kontext eine gängige

\footnotetext{
20 Vgl. Brill [1, S. 75].

21 ETH-Bibliothek Hochschularchiv Hs 87: 1495. Brief von Weiler aus Göttingen an Fiedler vom 20. April 1873.
} 
Bezeichnung für das (nicht materielle) Bild eines Objekts unter Reliefperspektive, folglich sind die fotografierten Modelle Modelle von Modellen - finden sich auf dem vorderen und hinteren Umschlag des Buches sowie pp. 142-143, wo auch deren Besonderheit erklärt wird. Man erkennt an diesen Beispielen sehr gut, dass das Modell nur aus einem Blickwinkel heraus passend erscheint.

Die Frage nach der Wichtigkeit des Modells als Vorlage für eine Serie, des Unterschiedes zwischen Original und Nachbildung (p. 20-22), und damit die des Konstrukteurs als Künstler war für mathematische Modelle von untergeordneter Wichtigkeit - keineswegs vergleichbar etwa mit der Kunstgeschichte. Oft wurde z.B. im Katalog von Brill/Schilling die Formulierung gewählt „unter der Leitung von“ und „hergestellt von“ etwa A. Brill und J. Bacharach (vgl. p. 214). ${ }^{22}$ Idee und Handwerk, mathematische Konzeption und konkrete Umsetzung wurden somit arbeitsteilig getrennt.

Was den kommerziellen Vertrieb von Modellen betrifft, so dominiert in „Mathematik mit Modellen“ natürlich der Verlag von Ludwig, der zum Leidwesen von Alexander Brill 1899 an Martin Schilling in Halle verkauft wurde. Offensichtlich gelang es ja Alexander mit den Modellen ein lukratives Geschäft für seinen Bruder zu organisieren; der Vertrieb begann 1879, er löste den durch Kreittmayr ab (vgl. p. 111-114). Bemerkenswert ist der internationale Erfolg der Brillschen Modelle, die sich in Europa und Nordamerika immer noch an vielen Stellen finden. Ein Exportschlager, würde man heute sagen. Im Buch werden noch zwei weitere Verlage erwähnt, zum einen der von Charles Delagrave in Paris, ursprünglich als kartographischer Verlag gegründet (1865), der die Modelle von Muret vermarktete (pp. 159-162), und die Lehrmittel-Anstalt Johann Ehrhardt \& Cie in Bensheim an der Bergstraße. Im Buch finden sich Abbildungen der gediegenen Holzmodelle dieser Firma, die noch in Tübingen vorhanden sind (pp. 201-208). Mit Ehrhardt wird eine andere Dimension der Modelle angesprochen, nämlich ihre Rolle für den technischen-gewerblichen Unterricht. Die weiten Betätigungsfelder der Fa. Ehrhardt, die von pädagogischen bis hin zu landwirtschaftlichen und technologischen Lehr- und Veranschaulichungsmitteln reichten, kann man der Titelseite ihres Katalogs von 1876 entnehmen (p. 205). Der wohl wichtigste Verlag dieser Ausrichtung befand sich in Brills Vaterstadt, nämlich der Verlag von Jakob Schröder, der ebenfalls Holzmodelle für den Geometrieunterricht vertrieb. Die von Ludwig Brill unterschiedene Ausrichtung der Verlage (,Anstalten“) von Ehrhardt und Schröder drückt sich in Bezeichnungen wie „Lehrmittelanstalt“ und „Unterrichtsmodelle“ deutlich aus. In Brills Vortrag von 1889 klingt die gewerblich-technische Bildung nur am Schluss ganz kurz an, wo er die seinerzeit sehr bekannten Modelle von Reuleaux zur Kinematik erwähnt. ${ }^{23}$ An einer technischen Hochschule hätten sich zahlreiche Anknüpfungspunkte geboten, an den Universitäten waren Technologen nicht (mehr) vertreten; vgl. die Arbeitsteilung zwischen Tübingen und Stuttgart.

An einer Stelle des Buches (p. 329) findet sich eine Wandtafel zum Thema Polyeder, die von G. Hessenberg, seit 1919 Professor in Tübingen, entworfen wurde. Wandtafeln wurden oft beim Unterrichten von Mathematik auf unterschiedlichen

22 Vgl. den Beitrag über Bacharach pp. 210-219.

23 Brill [1, S. 79]. 
Niveaus eingesetzt, sind aber leider nur selten erhalten geblieben und bislang wenig erforscht.

Zentrale Person im Buch „Mathematik mit Modellen“ ist natürlich Alexander von Brill. Dies zum einen, weil Brill ja die Tübinger Sammlung aufgebaut hat, um die es im Buch geht, zum andern aber auch, weil Brill zu einer wichtigen Persönlichkeit im Bereich mathematischer Modelle wurde. In München produzierte er oder ließ, teilweise in Kooperation mit F. Klein, zahlreiche Modelle produzieren, die dann als Urmodelle für solche dienten, die Bruder Ludwig vermöge seines Verlages verkaufte. Auch in Tübingen setzte er diese Aktivität, wenn auch reduziert, fort. Wenig erfährt die Leserin und der Leser über Brills Zeit vor seiner Berufung nach München, insbesondere über seine Zeit als Professor am Polytechnikum on Darmstadt (1869-1875). Brill schätzte die Hochschule seiner Vaterstadt wenig, er vermisste das wissenschaftliche Niveau, festzuhalten ist aber dennoch, dass Brill sechzehn Jahre an Polytechnika lehrte und arbeitete. Durch sein Doppelqualifikation als Architekt/ Ingenieur und Mathematiker war er für ein Polytechnikum bestens qualifiziert; die Konstruktion von Modellen, die an Polytechnika ubiquitär war $^{24}$, muss ihm sehr geläufig gewesen sein: Brill war insofern der polytechnischen Tradition ${ }^{25}$ eng verbunden.

Es gibt ein dunkles Kapitel in Brills Biographie, das aber keineswegs ausgespart wird im Buch, nämlich seine Annäherung an den Nationalsozialismus, insbesondere auch an dessen Antisemitismus (vgl. pp. 22-25). Im letzten Eintrag seines Rechenschaftsberichts „Aus meinem Leben“ heißt es laut seinem Biographen S. Finsterwalder, ehemals Assistent von Brill in Tübingen und Brills ,,alter Freund und Schüler“"26: „Aber ich möchte meiner Überzeugung doch einmal Ausdruck geben, daß mir das Vaterland in der Hand von Hitler die schönste Lösungen der Schwierigkeiten aller Art scheint, die Deutschland bis dahin bedroht haben. Möchte der Mann dem Volke noch lange, lange erhalten bleiben." 27 Neben den Modellen ist Brill vor allem als Koautor des mit Max Noether im Auftrag der DMV verfassten und 1892-93 publizierten Berichts über die Entwicklung der Theorie der algebraischen Funktionen bekannt geblieben. An verschiedenen Stellen seines Berichts sinniert Brill, der modern gesprochen recht kompetitiv gewesen zu sein scheint, über seinen Koautor, der ja bekanntlich Jude war - was damals hieß, der sich zur mosaischen Konfession bekannte: „Zu der Zusammenarbeit mit Noether. Es fehlt ihm, wie den Juden überhaupt, weder an durchdringendem Verstand noch an Arbeitskraft. Ihre Stärke ist gerade der kritisch-analysierende Verstand, der sich gerne in Gegenüberstellungen bewegt. Aber was Helmholtz in seiner neulichen Rede über Goethe das künstleri-

\footnotetext{
24 Man denke etwa an Modelle von Bauten, die Architekten ausführen.

25 Vgl. hierzu Volkert [15].

26 Brill [3, S. 83].

27 Finsterwalder [6, S. 663]. Finsterwalder spielt an mehreren Stellen seines Nachrufs durchaus mit affirmativem Unterton auf Brills Neigung zum Nationalsozialismus an. Im hier zu besprechenden Buch findet sich in der Einführung von Seidl p. 27 die Abbildung einer anderen letzten Seite von Brills Aufzeichnungen. Das dem Vf. dieser Besprechung vorliegende Exemplar stimmt weder mit den Aussagen Finsterwalders noch mit denen Seidls überein. Anscheinend gibt es mehrere Varianten dieses Berichtes. Die Meinige stammt aus dem Archiv der TU München. Der Vf. dankt Frau Hölzl (TUM Archiv) für ihre Hilfsbereitschaft und Unterstützung.
} 
sche Element in der wissenschaftlichen Tätigkeit nennt, scheint ihm zu fehlen: Die Erfindung neuer Gedankenketten, die Abrundung der Darstellung, das was auch die abstrakte Wissenschaft dem Menschen näher bringt, der Aufbau einer übersichtlichen Disposition und das Bildliche in der Rede. " 28 Es erscheint interessant zu klären, ob, und falls ja, wie der spätere Antisemitismus hier und in ähnlichen Betrachtungen Brills angelegt ist. Seidl weist auf einen weiteren scheinbar widersprüchlichen Aspekt von Brill Antisemitismus hin, nämlich den zwischen seiner Wertschätzung für Einzelpersonen wie Noether und der Abwertung des Judentums als Kollektiv. Übrigens findet Brill durchaus anerkennende Worte für Freund Noethers Tochter Emmy (p. 195 und 198); auch an der Bildung seiner eigenen Tochter zeigte er starkes Interesse.

Wie Brill sich selbst in Relation zu seiner Umwelt gesehen hat, wird aus der folgenden Passage seines Berichts deutlich ${ }^{29}$ :

Alex $^{30}$ berichtet über mich (....) ,,ich sei zu sehr Mathematiker, Stahl sei halb

Mathematiker, halb Mensch, ich aber sei bloß Mathematiker. ...

Und doch ist das Urteil ungerecht; wenigstens werden z.B. die Frauen finden, daß ich ihre Interessen kenne und viele derselben teile. Freilich Männer-Interessen gehen mir nur insofern nahe, als ich selbst dabei aktiv sein kann, d.h. als sie ein Feld betreffen, in welchem mir mitzuwirken möglich ist und Gelegenheit sich bietet.

Was ich anstreben müßte wäre 1) mich bei dem Anblick eines Menschen der Interessen zu erinnern, die mich etwa mit ihm verbinden könnten, und zuweilen selbst die Sprache darauf bringen, statt daß ich so, meist in meinen Kram versunken, bekannte Menschen wie vollkommen fremde behandle. 2) nicht zu lange bei einem Geprächsstoff verweilen, namentlich meine eigenen Äußerungen abzukürzen, aber deutlich und einmal sagen, was ich meine; 3) langsam reden. “

Damit möchte ich meine Bemerkungen zu diesem wirklich bemerkenswerten Buch und seiner Hauptfigur schließen. Es gehört in jede Fachbibliothek - schon allein um der schönen und informativen Fotos wegen. Aber natürlich nicht nur wegen dieser. Das einzige, was ich vermisst habe, sind Register. Die wären gewiss hilfreich, weil viele Themen an mehreren Stellen des Buches angesprochen werden. Schließen möchte ich mit einer für die mathematikhistorische Forschung interessanten Frage, die mir noch weitgehend offen zu sein scheint und sich beim Lesen des Buches geradezu aufdrängt: Warum erlebte das Interesse an materiellen Modellen etwa ab 1865 einen gewaltigen Aufschwung (,outburst“ (D. Rowe))?

Funding Open Access funding provided by Projekt DEAL.

\footnotetext{
28 Brill [3, S. 111-112], ähnlich allerdings noch ohne Verweis auf das Jüdische in Brills Nachruf auf „Freund“ Noether (Brill [2], 232). Brill war anscheinend mit seiner Rolle, seinem Anteil, am gemeinsamen Bericht unzufrieden. Er sah die kreativen Anteile bei Noether und bei sich die reproduktiven.

29 Brill [3, S. 138-139].

30 Alexander, der 1876 geborene älteste Sohn des Ehepaars Brill, Jurist und Präsident des Reichsausgleichsamtes.
} 
Open Access Dieser Artikel wird unter der Creative Commons Namensnennung 4.0 International Lizenz veröffentlicht, welche die Nutzung, Vervielfältigung, Bearbeitung, Verbreitung und Wiedergabe in jeglichem Medium und Format erlaubt, sofern Sie den/die ursprünglichen Autor(en) und die Quelle ordnungsgemäß nennen, einen Link zur Creative Commons Lizenz beifügen und angeben, ob Änderungen vorgenommen wurden.

Die in diesem Artikel enthaltenen Bilder und sonstiges Drittmaterial unterliegen ebenfalls der genannten Creative Commons Lizenz, sofern sich aus der Abbildungslegende nichts anderes ergibt. Sofern das betreffende Material nicht unter der genannten Creative Commons Lizenz steht und die betreffende Handlung nicht nach gesetzlichen Vorschriften erlaubt ist, ist für die oben aufgeführten Weiterverwendungen des Materials die Einwilligung des jeweiligen Rechteinhabers einzuholen.

Weitere Details zur Lizenz entnehmen Sie bitte der Lizenzinformation auf http://creativecommons.org/ licenses/by/4.0/deed.de.

\section{Literatur}

1. Brill, A.: Ueber die Modellsammlung des mathematischen Seminars der Universität Tübingen. Math.Naturwiss. Mitteilungen 2, 69-80 (1889)

2. Brill, A. von: Max Noether. Jahresbericht Dtsch. Math. Ver. 32, 211-234 (1923)

3. Brill, A. von: Aus meinem Leben (Typoskript 1928). Aus dem Archiv der Technischen Universität München, Bestand TUM Archiv KS

4. Etwein, F.: Ein Leben für die Polyeder - der Oberlehrer Max Brückner und seine Modelle. Math Semesterber 66, 15-30 (2019)

5. Fiedler, W.: Stereoskopische Photographien des Modelles einer Fläche dritter Ordnung mit 27 reellen Geraden. Z. Math. Phys. 14, 32-34 (1869). Litteraturzeitung

6. Finsterwalder, S.: Alexander v. Brill. Ein Lebensbild. Math. Ann. 112, 653-663 (1937)

7. Geppert, A.C.T.: Welttheater: Die Geschichte des europäischen Ausstellungwesens im 19. Und 20. Jahrhundert. Neue Polit. Lit. 47, 10-61 (2002)

8. Klinger, K.: Zwischen Gelehrtenwissen und handwerklicher Praxis. Fink, Paderborn (2014)

9. König, W.: Künstler und Strichezieher. Suhrkamp, Frankfurt a. M. (1999)

10. Labs, O.: Straight lines on models of curved surfaces. Math. Intell. 39(2), 15-20 (2017)

11. Lê, F.: Entre géométrie et théorie des substitutions: une étude de cas autour des vingt-sept droites d'une surface cubique. Confluentes Math. 5, 23-71 (2013)

12. Lipsmeier, A.: Technik und Schule. Steiner, Wiesbaden (1971)

13. Rowe, D.: Mathematical models as artefacts for research: Felix Klein and the case of Kummer surfaces. Math Semesterber 60, 1-24 (2013)

14. Tobies, R., Volkert, K.: Mathematik auf den Versammlungen der Gesellschaft deutscher Naturforscher und Ärzte 1843-1890. Wissenschaftliche Buchgesellschaft, Stuttgart (1998)

15. Volkert, K.: Mathematische Modelle und die polytechnische Tradition. Siegener Beiträge Zur Gesch. Philos. Math. 10, 161-202 (2018) 